BMJ Open Sport \& Exercise Medicine

\title{
Effects of aerobic exercise on thyroid hormonal change responses among adolescents with intellectual disabilities
}

\author{
Kefelegn Zenebe Altaye, ${ }^{1}$ Soumitra Mondal, ${ }^{2}$ Kesatie Legesse, ${ }^{2}$ \\ Mahmud Abdulkedir ${ }^{3}$
}

To cite: Altaye KZ, Mondal S, Legesse $\mathrm{K}$, et al. Effects of aerobic exercise on thyroid hormonal change responses among adolescents with intellectual disabilities. BMJ Open Sport \& Exercise Medicine 2019;5:e000524. doi:10.1136/ bmjsem-2019-000524

Accepted 16 May 2019

\section{ABSTRACT}

Objective We aimed to investigate the impact of a 16-week aerobic exercise programme on the changes in the plasma level concentration of thyroid hormones in adolescents with intellectual disabilities.

Methodology Using purposive sampling, a total of 36 adolescents with intellectual disabilities were selected to participate in the study. The training programme consisted of 16 weeks of moderate-intensity aerobic exercises at an intensity of $45-75$ hours. These exercises were performed in three sessions a week, and each session took 30-45 min: 10 min warm-up, 15-30 min main aerobic workout and 5 min cool-down exercises. The plasma levels of triiodothyronine (T3) and tetraiodothyronine (T4) and of thyroid stimulating hormone were measured before and after 16 weeks of aerobic exercise intervention.

Results After 16 weeks of intervention, a significant change was observed in the plasma level concentration of thyroid (T3 and T4) and thyroid stimulating hormones $(p<0.05)$ in the group treated with aerobic exercise.

Conclusion We concluded that aerobic exercise had an impact on the change in the plasma level concentration of thyroid and thyroid stimulating hormones in adolescents with intellectual disabilities.

\section{INTRODUCTION}

Intellectual disability (ID) is characterised by significant limitations in cognitive functioning, adaptive behaviour, and conceptual, social and practical skills. In addition, when compared with their typically developed peers, persons with ID are more likely to be obese, less likely to be physically active and are twice as likely to develop a chronic disease. ${ }^{1}$ Also, compared with their normal peers, they are more limited in how well and how quickly they can learn, and score the lowest and more likely to repeat each class level. ${ }^{2}$ The percentage of individuals with ID who completed primary education is significantly lower than persons without disabilities, ${ }^{3}$ and often they have cognitive problems associated with carrying out exercises. ${ }^{4}$ The worldwide and Ethiopian prevalence of ID is $1.3 \% .^{5}$ ID is associated with lower levels of thyroid

\section{What is the new finding?}

- Aerobic exercise increases the concentration of triiodothyronine and tetraiodothyronine in the blood, which have an exciters effect on the central nervous system, leading to academic improvement and success of adolescents with intellectual disabilities.

- Aerobic exercise can decrease the concentration of thyroid stimulating hormone in the blood of adolescents with intellectual disabilities.

- Aerobic exercise can maintain the equilibrium in thyroid and thyroid stimulating hormone homeostasis in adolescents with intellectual disabilities.

\section{What is already known?}

Aerobic physical exercise has a great impact on energy expenditure, reduces body fat mass and improves the lung function of individuals with intellectual disabilities.

- Aerobic physical exercise increases the metabolism of lactic and fatty acids and affects changes in hormonal concentrations in adolescents with intellectual disabilities.

- Aerobic physical exercise plays a significant role in increasing brain function, which is linked to cognitive development and academic achievement in adolescents with intellectual disabilities.

hormones. In relation to these, thyroid hormone deficiency during fetal and postnatal development may cause retarded brain maturation, intellectual deficits, and in some cases neurological impairments. ${ }^{6}$ However, proportional secretion of tetraiodothyronine (T4) and triiodothyronine (T3) accelerates growth and stimulates activities in the nervous system. ${ }^{7}$ Therefore, thyroid hormones are important for normal brain and nervous system development and function. ${ }^{8}$

Previous studies reported that aerobic exercise increases total serum T3 and T4. ${ }^{9} 10$ Consequently, a moderate-intensity level of exercise can increase $\mathrm{T} 4$ concentration in the blood. ${ }^{11}$ Aerobic exercise has been 
associated with a progressive decrease or an improvement in serum thyroid stimulating hormone (TSH) ${ }^{12}$ On the contrary, 12 weeks of aerobic exercise had shown an insignificant change in the plasma level of TSH, T3 and T4 hormones among sedentary women. These hormonal fluctuations are dependent on the intensity, duration and type of exercise and on the individual's age, ${ }^{13}$ and still contradictory results were seen on the effects of aerobic exercise on thyroid hormonal change responses. ${ }^{14}$ Little information has been seen on the effects of aerobic exercises on thyroid hormone change responses in adolescents with ID.

Therefore, we designed the present study to investigate the effects of 16 weeks of aerobic exercises on hormonal change concentration in adolescents with ID. This was done using quasi-experimental research design and using T3, T4 and TSH as outcome markers. Based on the findings of the study, the lifestyle and participation of adolescents with ID in social and economic activities might be enhanced to support their family and their country at large. The study may also serve as a springboard for those who are interested to conduct further similar research in the area, especially by including diet as one of the variables, among others.

\section{MATERIALS AND METHODS Study design}

A quasi-experimental design was employed to investigate the effects of 16 weeks of aerobic exercise on thyroid hormonal change responses in adolescents with ID in Dessie-Kombolcha.

\section{Sample and sampling technique}

The study has two groups: experimental and control groups. The participants were free from epilepsy and autism disorders. The total number of the population was 72 , and in the current study 36 of them, which were $50 \%$ of the total population, were purposively taken as a sample and were randomly selected and placed in the experimental and control groups. All participants were randomly divided into two groups, control and experimental groups, with 18 participants each. After explaining the purposes of the study and the privacy of information to the participants and their guardians, they were completely satisfied to cooperate and signed a consent agreement to participate in this study. The participants of the experimental group were invited to take orientation about how to execute planned aerobic exercises before the first day of the actual training period, while the participants of the control group committed to not participate in any exercise except their formal schools physical exercise periods during the 16-week period of the study.

\section{Blood analysis}

In order to measure the plasma level of TSH and thyroid hormones (T3 and T4), blood samples were drawn from adolescents 48 hours before the first training session and 48 hours after the last training session. Samples of blood specimen were collected in sitting position by qualified laboratory technicians. Six millilitres of venous blood were drawn from each participant's vein pretest and posttest. The blood samples were drawn into tubes containing an anticoagulant. All blood samples were preserved in a frozen condition at $-20^{\circ} \mathrm{C}$ and were conserved until analysis. All variables were measured by ELISA using Autobio laboratory kits.

\section{Training protocol}

The training programme consisted of 16 weeks of moderate-intensity aerobic exercises and was conducted after receiving parental consent. These exercises were performed three sessions a week, and each session took 30-45 min. Each session consisted of $10 \mathrm{~min}$ of warm-up exercises, 15-30 min of main aerobic workout and $5 \mathrm{~min}$ cool-down exercises. Study participants and their parents / guardians have a complete right to reduce and stop the exercise, and it is their obligation to inform the fitness training programme personnel should any symptoms develop. Therefore the fitness trainer can reduce or stop the exercise programme should any symptoms develop and will indicate that this was done for the benefit and safety of the participants. Moreover the participants have an absolute right to withdraw from the programme by themselves or with the help of their parents/guardians.

\section{Statistical analysis}

In this study, independent t-test was used to compare intergroup variations between experimental and control groups on the effects of 16 weeks of aerobic exercise on thyroid hormone change responses in adolescents with ID. Significance level was considered at $\mathrm{p} \leq 0.05$.

\section{RESULTS}

Thirty-six adolescents with ID were studied. Table 1 shows the mean and SD of age, height and mass of adolescents with ID. For adolescents with ID treated with aerobic exercise, the mean (SD) age, height (in metre) and mass (in kilogram) were 14.44 (1.199), $1.50(0.042)$ and 49 $(\mathrm{SD}=5.89)$, respectively, and for the control group the mean (SD) age, height and mass were 14.389 (1.145), $1.49(0.046)$ and $48.16(6.148)$, respectively.

Table 2 shows the mean and SD score in hormonal concentration change responses between the experimental and control groups using independent t-test in T3 $(, p=0.0005)$, the mean and SD score in hormonal change

Table 1 Demographic characteristics of adolescents with intellectual disabilities in the aerobic and control groups

\begin{tabular}{lccccc}
\hline & \multicolumn{2}{c}{ Aerobic group } & & \multicolumn{2}{c}{ Control group } \\
\cline { 2 - 3 } \cline { 5 - 6 } Variables & Mean & SD & & Mean & SD \\
\hline Age (years) & 14.44 & 1.199 & & 14.389 & 1.145 \\
Height (metre) & 1.50 & 0.042 & & 1.49 & 0.046 \\
Mass $(\mathrm{kg})$ & 49 & 5.89 & & 48.16 & 6.148 \\
\hline
\end{tabular}


Table 2 Average and SD of thyroid hormone change responses in adolescents with intellectual disabilities between the control and experimental groups using independent t-test

\begin{tabular}{llllcll}
\hline $\mathbf{s} / \mathbf{n}$ & Variables & Group & Mean \pm SD & t value & df & P value \\
\hline 1 & T3 & Aerobic & $1.713 \pm 0.224$ & 4.953 & 34 & $0.005^{*}$ \\
& & $\begin{array}{l}\text { Control } \\
1.421 \pm 0.11\end{array}$ & & & \\
2 & T4 & Aerobic & $8.349 \pm 1.043$ & 5.253 & 34 & $0.0001^{*}$ \\
& & Control & $6.617 \pm 0.931$ & & & \\
3 & \multirow{2}{*}{ TSH } & Aerobic & $1.097 \pm 0.157$ & -2.971 & 34 & $0.0001^{*}$ \\
& & Control & $1.413 \pm 0.423$ & & & \\
\hline
\end{tabular}

*The mean difference is significant at $\mathrm{p}<0.05$.

T3, triiodothyronine; T4, tetraiodothyronine; $\mathrm{TSH}$, thyroid stimulating hormone; $\mathrm{s} / \mathrm{n}$, serial number.

responses between the experimental and control groups using independent $\mathrm{t}$-test in $\mathrm{T} 4(\mathrm{t}=5.253, \mathrm{p}=0.0001)$, and the mean and SD score in hormonal change responses between the experimental and control groups using independent t-test in TSH $(\mathrm{t}=-2.971, \mathrm{p}=0.0001)$.

\section{DISCUSSION}

We aimed to investigate the effects of aerobic exercise on thyroid and thyroid stimulating hormonal concentration change responses in adolescents with ID. Based on the findings of the study, the aerobic exercise intervention had shown a significant change response in the plasma levels of TSH and thyroid hormones (T3, T4) when compared with the control group.

The results of this study are consistent with previous studies $^{9-1215}$ that reported serum TSH was found significantly decreased and T3 and T4 significantly increased with aerobic exercise intervention. Acute aerobic exercise performed at moderate intensity induced significant increases in total serum T3 and T4. This confirms that aerobic exercise may enhance the thyroid hormone concentration in the serum. However, the present study was in contrast to previous studies 81617 that reported aerobic exercise had no significant effect on thyroid hormone concentration. These variations might be due to the specific characteristics of students with ID. Individuals with ID have thyroid hormone deficiency ${ }^{18}$ and have lower aerobic fitness levels compared with their equivalent able peers. ${ }^{1219}$ Thus, ${ }^{20}$ confirmed that all these can be improved by physical exercise.

In this study, significant improvements in T3, T4 and $\mathrm{TSH}$ in response to aerobic exercises might be the specific characteristics of those adolescents with ID and the methodological differences in the type, intensity and frequency of the training protocol. Thus, the researcher believed that 4 months of aerobic intervention programme is possibly too long to achieve equilibrium in thyroid and thyroid stimulating hormone homeostasis in adolescents with ID.

Aerobic exercise has a number of physiological and psychological impacts. It has a great impact on the growth of new blood vessels in the brain, which increases cognitive impairments, ${ }^{21}$ improves lung function in ID,${ }^{22}$ increases the efficiency of aerobic endurance, ${ }^{23}$ and increases T3 and T4 levels, ${ }^{11}$ which have an effect on the exciter nerve cells of the central nervous system, ${ }^{24}$ leading to academic improvement and success of students. ${ }^{25}$ Thus, aerobic physical exercise has a therapeutic effect, and parents can use it as a treatment to minimise the physiological causes of cognitive impairment and improve the lifestyle of adolescents with ID. Thus, adolescents with ID should be motivated to undertake regular aerobic exercises to enhance their participation in educational, social and economic activities. An investigation on thyroid and thyroid stimulating hormonal change responses in relation to adolescents with ID, which have been uncovered by other researchers, should be considered a strength of this study. The results of the study can be used as a springboard for those who are interested to conduct further similar research work in this area, especially by including diet as one of the variables, among others.

\section{Limitations}

The limitations of this study were the problem of working with adolescents with ID and the lack of timely presence of the study participants during the training sessions.

\section{CONCLUSION}

We concluded that aerobic exercises of moderate intensity are an efficient method to change the plasma concentration of thyroid and thyroid stimulating hormones in adolescents with ID.

Acknowledgements The authors would like to acknowledge the study participants and their parents/guardians. All of their teachers, training coaches and school directors who helped in this study are greatly appreciated.

Contributors All authors made great contributions to the completion of the study. MA participated in the methodology and data analysis. KL participated in the sequence alignment and interpretation of data, and drafted the manuscript. KZA contributed to writing the original draft and preparation. SM participated in writing and review, sequence alignment, and editing of the manuscript.

Funding The authors have not declared a specific grant for this research from any funding agency in the public, commercial or not-for-profit sectors.

Competing interests None declared.

Patient consent for publication Not required.

Ethics approval The experiment complied with the laws of ethical clearance committee (HRERC), and the College of Health Science, Mekelle University approved the study (ERC0775/2016). Informed consent was obtained from all participants and their parents/guardians before testing and commencing the study. The participants and their parents/guardians signed and confirmed their voluntary agreement to full participation in this scientific training session project. With regard to ethical conditions of the research, the researchers made sure that no one was affected by this scientific study. Only $6 \mathrm{~mL}$ of blood was taken from the study participants during each of the pretests and post-tests.

Provenance and peer review Not commissioned; externally peer reviewed.

Data availability statement Data are available upon reasonable request.

Open access This is an open access article distributed in accordance with the Creative Commons Attribution Non Commercial (CC BY-NC 4.0) license, which permits others to distribute, remix, adapt, build upon this work non-commercially, and license their derivative works on different terms, provided the original work is properly cited, appropriate credit is given, any changes made indicated, and the use is non-commercial. See: http://creativecommons.org/licenses/by-nc/4.0/. 


\section{REFERENCES}

1. Fernhall B. Physical fitness and exercise training of individuals with mental retardation. MedSci Sports Exerc 1993;25:442-50.

2. Axuter D, Pyfer J, Huetting C. Principles and methods of adapted physical education and recreation. 3th edn. Mosby year book, 2005.

3. Posarac A, Mitra S, Vick B. Disability and poverty in developing countries potentials. J Cogn Neurosci 2011;3:151-65.

4. Hayakawa K, Kobayashi K. Physical and motor skill training for children with intellectual disabilities. Percept Mot Skills 2011;112:573-80.

5. Iqbal M, Baig MA, Bhinder MA, et al. Factors causing mental retardation. AJSC 2016;5:28-37.

6. Bernal J, Anawalt B, Boyce A. Thyroid hormones in brain development and function, 2015.

7. Sherwood L. Human physiology. In: Arbogast M, Hopperstead K, Lee J, eds. From cell to system. 6th edn. China, 2007.

8. Rahimi E, Zadeh MY, Boostani AM. The effect of resistance training on thyroid hormones. Euro J Exp Bio 2013;3:443-7.

9. Ciloglu F, Peker I, Rehlivan A, et al. Exercise intensity and its effects on thyroid hormones. Neuro Endocrinol Lett 2005;6:830-4.

10. Klubo-Gwiezdzinska J, Bernet VJ, Wartofsky L. Exercise and thyroid function. J Sport Exerc Psychol 2013:85-119.

11. Barari RA. Endurance training and ginger supplement on $\mathrm{TSH}, \mathrm{T} 3$, T4 and testosterone and cortisol hormone in obese men. Iran $J$ Basic Med Sci 2016:3:96-103.

12. Krotkiewski M, Sjostrom L, Sullivan L, et al. The effect of acute and chronic exercise on thyroid hormones in obesity. Acta Med Scand 1984;216:269-75

13. Lencu C, Nicula R, Lotrean MR. Hormonal response to physical exercise Răspunsul hormonal la efortul fizic:Palestrica of the third millennium - Civilization and Sport. 2016;17:56-60.
14. Arkadar R, Rosa MR, Moretti G. Physiological change of exercise of thermo genesis, thyroid homeostasis and inflammation. Arch Endocrinol Metab 2016;3.

15. Bansal A, Kaushik A, Singh CM, et al. The effect of regular physical exercise on the thyroid function of treated hypothyroid patients: an interventional study at a tertiary care center in Bastar region of India. Arch Med Health Sci 2015;3:244-6.

16. Onsori M, Galdari M. Effects of 12 weeks aerobic exercise on plasma level of TSH and thyroid hormones in sedentary women. Eur J Sport Sci 2015;4:45-9.

17. Sullo A, Brizzi G, Maffulli N. Deiodinating activity in the brown adipose tissue of rats following short cold exposure after strenuous exercise. Physiol Behav 2003;80:399-403.

18. Rivas M, Naranjo JR. Thyroid hormones, learning and memory. Genes Brain Behav 2007;6(Suppl 1):40-4

19. Davies KJ, Packer L, Brooks GA. Biochemical adaptation of mitochondria, muscle, and whole-animal respiration to endurance training: archsbiochem Biophys 1981;209:539-44.

20. Gillespie M. Cardiovascular fitness of young Canadian children with and without mental retardation. Educ Train Dev Disabil 2003:38:296-301.

21. Pang MYC, Eng JJ, Dawson AS, et al. The use of aerobic exercise training in improving aerobic capacity in individuals with stroke: a meta-analysis. Clin Rehabil 2006;20:97-111.

22. Godman H. Regular exercise changes the brain to improve memory, thinking skills. Harvard health letter 2018.

23. Ozmen T, Ryildirim NU, Yuktasir B, et al. Effects of school-based cardiovascular-fitness training in children with mental retardation. Pediatr Exerc Sci 2007;19:171-8.

24. Prentice WEMalinee V, Red S, eds. Rehabilitation techniques in sports medicine. USA: EdwardG Bartell, 1999.

25. Taras H, Howard T. Physical activity and student performance at school. J Sch Health 2005;75:214-8. 